\title{
Fixação intramedular flexível para fraturas diafisárias dos ossos do antebraço em crianças*
}

\section{Intramedullary Flexible Nailing for Diaphyseal Fractures of Forearm Bones in Children}

\author{
Balakrishnan M. Acharya ${ }^{1}$ Pramod Devkota ${ }^{1}{ }^{1}$ Abhishek K. Thakur ${ }^{1} \quad$ Bidur Gyawali $^{1}$ \\ 1 Departamento de Ortopedia e Cirurgia do Trauma, Patan Academy \\ of Health Sciences, Patan Hospital, Lalitpur, Nepal \\ Rev Bras Ortop 2019;54:503-508. \\ Endereço para correspondência Pramod Devkota, MBBS, MS (Ortho), \\ Department of Orthopaedics and Trauma Surgery, Patan Academy of \\ Health Sciences, Patan Hospital, Lalitpur, Nepal \\ (e-mail: devkotap@gmail.com).
}

\section{Resumo \\ Palavras-chave \\ - crianças \\ - traumatismos do antebraço \\ - fixação intramedular de fraturas \\ - fraturas da ulna \\ - fraturas do rádio \\ - pinos ortopédicos}

Objetivos A maioria das fraturas dos ossos do antebraço em crianças é tratada com sucesso de forma conservadora com redução fechada e imobilização gessada. No entanto, alguns pacientes podem necessitar de manipulação adicional da fratura ou intervenção cirúrgica devido a angulações residuais. O presente estudo avalia o resultado clinico e radiológico do tratamento de fraturas com desvio do antebraço em crianças fixadas com haste elástica intramedular de titânio.

Métodos Um total de 31 pacientes com idades entre 7 e 15 anos com fraturas com desvio do antebraço foram submetidos a haste elástica de titânio flexível. Os pacientes foram acompanhados por um período médio de 8,51 meses (variação: 6-12 meses) e avaliados quanto aos resultados funcionais pelos critérios de Price e radiológicos.

Resultados Dos 31 pacientes, 21 foram submetidos a redução fechada e 10 necessitaram de abertura mínima do local da fratura durante a redução. Um total de 29 pacientes tiveram excelentes resultados com arco de movimento normal, e 2 pacientes apresentaram bons resultados. Em todos os pacientes, a consolidação ocorreu em um tempo médio de 7,9 semanas. Cinco pacientes tiveram complicações menores, como irritação da pele sobre a haste proeminente $(n=2)$, infecção superficial do local de inserção no rádio $(n=2)$ e recuo da haste ulnar $(n=1)$, que exigiu remoção precoce.

Conclusão A haste flexível é um método eficiente para tratamento da fratura do antebraço em crianças, permitindo mobilização precoce e retorno às atividades normais dos pacientes, com baixas e tratáveis complicações.

Objectives Most of the fractures of the bones of the forearm in children are successfully treated conservatively with closed reduction and casting. The outcomes
Trabalho realizado no Departamento de Ortopedia e Cirurgia do Trauma, Patan Academy of Health Sciences, Patan Hospital, Lalitpur, Nepal. recebido

25 de Dezembro de 2018 aceito

13 de Maio de 2019
DOI https://doi.org/

10.1055/s-0039-1693742. ISSN 0102-3616.
Copyright $\odot 2019$ by Sociedade Brasileira License terms de Ortopedia e Traumatologia. Published by Thieme Revnter Publicações Ltda, Rio de Janeiro, Brazil 


\section{Keywords \\ - children \\ - forearm injuries \\ - fracture fixation intramedullary \\ - ulna fractures \\ - radius fractures \\ - bone nails}

remain variable and the patients may require additional fracture manipulation or formal surgical intervention due to residual angulations. The present study assesses the radiological and functional outcomes of treating displaced forearm fractures in children with intramedullary flexible titanium elastic nailing.

Methods A total of 31 patients aged between 7 and 15 years old with displaced forearm fractures underwent flexible titanium elastic nailing. The patients were followed-up for a mean period of 8.51 months (range: 6-12 months) and were assessed for radiological and functional outcomes. The Price criteria were used to access the functional outcome.

Results Out of 31 patients, 21 patients underwent closed reduction, and 10 required a minimal opening of the fracture site during reduction. A total of 29 patients had excellent results with normal forearm and elbow range of motion (ROM), and 2 patients had good results. In all patients, good radiological union was seen at an average time of 7.9 weeks. Five patients had minor complications, such as skin irritation over the prominent ulnar nail $(n=2)$, superficial nail insertion site infection $(n=2)$, and backing out of the ulnar nail $(n=1)$, requiring early removal.

Conclusion Flexible nailing is an efficient application of internal fixation for shaft fractures of both bones of the forearm in children, enabling early mobilization and return to the normal activities of the patients, with low and manageable complications.

\section{Introdução}

As fraturas do antebraço são as fraturas mais comuns de ossos longos em crianças, compreendendo a $\sim 40 \%$ de todas as fraturas pediátricas. ${ }^{1}$ Embora as fraturas dos ossos do antebraço sejam tratadas com sucesso de forma conservadora, os resultados permanecem variáveis e, posteriormente, alguns casos podem exigir manipulação adicional da fratura ou intervenção cirúrgica formal devido a angulações residuais. ${ }^{2-6}$ Estudos anteriores mostraram que a falha do tratamento não cirúrgico das fraturas mediodiafisárias em populações pediátricas varia entre 39 e $64 \%{ }^{3}$ As alternativas incluem remanipulação fechada e imobilização, pinos e gesso, redução fechada ou miniaberta com haste intramedular (IM) e redução aberta com fixação interna (RAFI) usando placas e parafusos. ${ }^{2}$

Resultados bons a excelentes foram relatados em várias séries, levando ao entusiasmo generalizado com a fixação IM. ${ }^{4,7}$ Vários dispositivos IMs, como o Pino de Rush, os fios de Kirschner (fios K), ou as hastes de Ender, estão disponíveis para o tratamento dessas fraturas, mas as hastes elásticas de titânio ganharam grande popularidade devido à sua maior elasticidade e melhor estabilidade rotacional em relação aos outros pinos de aço. 8,9

A intervenção cirúrgica com haste flexível tem sido aplicada cada vez mais também em nosso contexto. 0 presente estudo foi realizado para avaliar o resultado radiológico e funcional, assim como as complicações associadas às fraturas dos ossos do antebraço tratadas com hastes elásticas de titânio em crianças (-Fig. $\mathbf{1}$ ).

\section{Materiais e Métodos}

Este é um estudo observacional retrospectivo realizado no Departamento de Ortopedia e Cirurgia do Trauma do Patan
Hospital, Patan Academy of Health Sciences (PAHS), Lalitpur, Nepal, entre março de 2015 e fevereiro de 2017. O presente estudo foi aprovado pelo Institutional Review Committee (IRC) da Patan Academy of Health Sciences, Patan Hospital, e um consentimento fornecido por escrito foi obtido de todos os pais dos pacientes. Os critérios de inclusão foram crianças $<15$ anos, de ambos os sexos, com fraturas diafisárias fechadas ou abertas do tipo $\mathrm{I}^{10}$ no rádio ou na ulna após insucesso da redução fechada e fraturas completamente deslocadas ou completas de ambos os ossos com angulação $>30^{\circ}$ antes da redução. Os critérios de exclusão foram fraturas abertas que não do tipo I, ${ }^{10}$ fraturas associadas a lesões neurovasculares, fraturas com mais de uma semana, fraturas em galho verde, fratura de Monteggia, e fraturas patológicas. Todas as fraturas dos ossos do antebraço foram fixadas com hastes elásticas de titânio para o rádio e a ulna usando técnica e princípios cirúrgicos padrão, usando fluoroscópio de arco em C. Gesso acima do cotovelo foi aplicado por 6 semanas. Os casos tiveram acompanhamento em 2 semanas, 6 semanas, 12 semanas, 24 semanas, e 9 meses ou 1 ano após a cirurgia. Os resultados clínicos foram classificados no acompanhamento final de acordo com o sistema descrito por Price. ${ }^{11}$ Os critérios de Price são amplamente utilizados na literatura para avaliar o resultado estético e funcional das fraturas ósseas do antebraço das crianças. $O$ resultado é considerado excelente quando não há queixa nas atividades extenuantes e/ou $<10^{\circ}$ de perda de rotação do antebraço; o resultado é considerado bom quando há leve queixa nas atividades extenuantes e/ou entre $11^{\circ}$ e $30^{\circ}$ de perda de rotação do antebraço. O resultado é considerado razoável quando há leves queixas subjetivas durante as atividades diárias e/ou entre $31^{\circ}$ e $90^{\circ}$ de perda de rotação do antebraço; e todos os restantes são considerados resultados pobres. A análise estatística foi realizada no software IBM SPSS Statistics for Windows, Versão 20.0 (IBM Corp., Armonk, NY, EUA). 


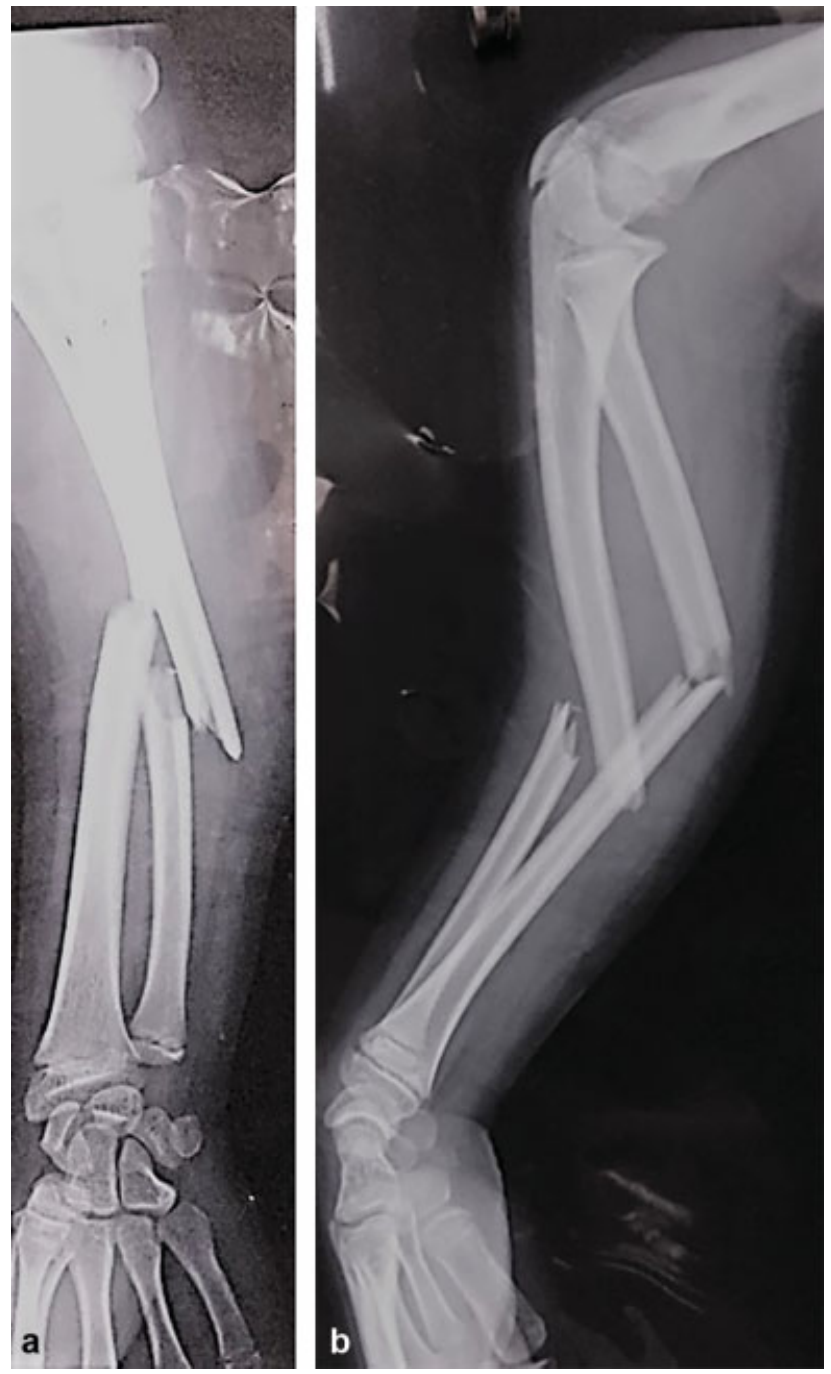

Fig. 1 (a) Visão anteroposterior (AP) e 1 (b) visão lateral de um menino de 10 anos que sofreu fratura do antebraço do lado direito.

\section{Técnica Cirúrgica}

A cirurgia foi realizada sob um fluoroscópio de arco em C, utilizando anestesia geral ou bloqueio regional, utilizando a técnica operatória descrita por Lascombes et al. ${ }^{12}$ Foi feita uma incisão na pele $1 \mathrm{~cm}$ proximal à fise radial distal ao longo do sulco do punho. A haste foi ligeiramente dobrada a 1 polegada da ponta para facilitar a inserção, caso a passagem através do local da fratura fosse difícil. A haste radial IM foi introduzida de forma retrógrada, proximal ao tubérculo de Lister. As precauções ideais foram tomadas para proteger os tendões extensores e o nervo cutâneo radial superficial. Quando não foi possível inserir a haste sem incisão, uma pequena incisão foi feita no local da fratura, expondo as extremidades da fratura para que a redução fosse realizada. A haste IM ulnar foi introduzida de forma anterógrada através de uma incisão longitudinal feita $1 \mathrm{~cm}$ distal à apófise do olecrano. Após a inserção da haste IM, a limitação da pronação do antebraço e da supinação foi examinada no intraoperatório para evitar potencial má união rotacional. O comprimento da haste foi medido da epífise proximal à distal sob o fluoroscópio de braço em C, e o diâmetro da haste variou entre 2 e $3 \mathrm{~mm}$, dependendo do diâmetro da cavidade medular no ponto do istmo. As extremidades distais das hastes foram enterradas sob a pele em todos os casos.

\section{Resultados}

Foram incluídas no estudo um total de 31 crianças com fraturas do antebraço, 22 (71,96\%) do sexo masculino e 9 (29,03\%) do sexo feminino. Havia um total de 55 pacientes que tiveram ambas as fraturas do antebraço durante o período compreendido pelo estudo, e apenas 43 preencheram os critérios de inclusão. Contudo, devido a várias razões, como restrições financeiras e de tempo dos pais, apenas 31 pacientes foram operados por fixação IM de haste flexível. A média de idade dos pacientes foi de 12,90 anos (variação: 7 a 15 anos). Um total de $25(80,64 \%)$ pacientes apresentava fraturas do antebraço do lado direito, e 6 (19,35\%) do lado esquerdo.

Um total de $28(90,32 \%)$ pacientes teve fraturas fechadas, e 3 $(9,67 \%)$ pacientes tiveram fraturas expostas do tipo $\mathrm{I}^{10} \mathrm{O}$ padrão de fratura foi transversal em $19(61,29 \%)$ pacientes, oblíquo em 8 (25,8\%) pacientes, cominutivo em 3 (9,68\%) pacientes, e em espiral em 1 (3,22\%). A localização da fratura foi o terço médio em 22 (70,97\%) pacientes, o terço proximal em $6(19,35 \%)$ pacientes, e o terço distal em $3(9,67 \%)$ pacientes. Uma abertura mínima do local da fratura durante a redução foi necessária em 10 (32,23\%) pacientes, dos quais 6 (19,35\%) necessitaram somente na ulna, $2(6,45 \%)$ pacientes necessitaram apenas no rádio, e $2(6,45 \%)$ pacientes necessitaram tanto no rádio quanto na ulna.

Os pacientes foram acompanhados por um tempo médio de 8,51 meses (variação: de 6 a 12 meses). 0 tempo médio para a consolidação da fratura, definida como a presença de calos ósseos ${ }^{13}$ em vistas anteroposterior (AP) e lateral das radiografias, foi de 7,9 semanas (intervalo: de 6 a 12 semanas). Segundo os critérios de Price, resultados excelentes foram obtidos em 29 (93,55\%) pacientes, e bons em 2 (6,45\%) pacientes. Entre os pacientes com bons resultados, ambos tiveram restrição de supinação do antebraço de $\sim 20^{\circ}$ no último acompanhamento. Dos 31 pacientes, 5 (16,12\%) tiveram complicações menores, como irritação da pele devida a material proeminente na ulna em $2(6,45 \%)$ pacientes, infecção superficial do local da inserção (no rádio) em 2 $(6,45 \%)$ pacientes, e recuo da haste ulnar exigindo remoção precoce em 1 (3,22\%) paciente. Complicações maiores, como discrepância no comprimento do membro, afetando as funções das extremidades, deformidade angular ou rotacional, sinostose, ou movimento restrito do cotovelo, não foram encontradas.

A remoção dos implantes foi feita em 10 pacientes (32,3\%), e os casos restantes ainda estão em acompanhamento. 0 tempo médio de remoção foi de 7 meses (variação: de 6 a 10 meses) (-Fig. 2).

\section{Discussão}

A maioria das fraturas pediátricas do antebraço pode ser tratada de forma não cirúrgica por redução fechada e imobilização. ${ }^{13-16}$ As fraturas diafisárias e aquelas que são proximais não se 


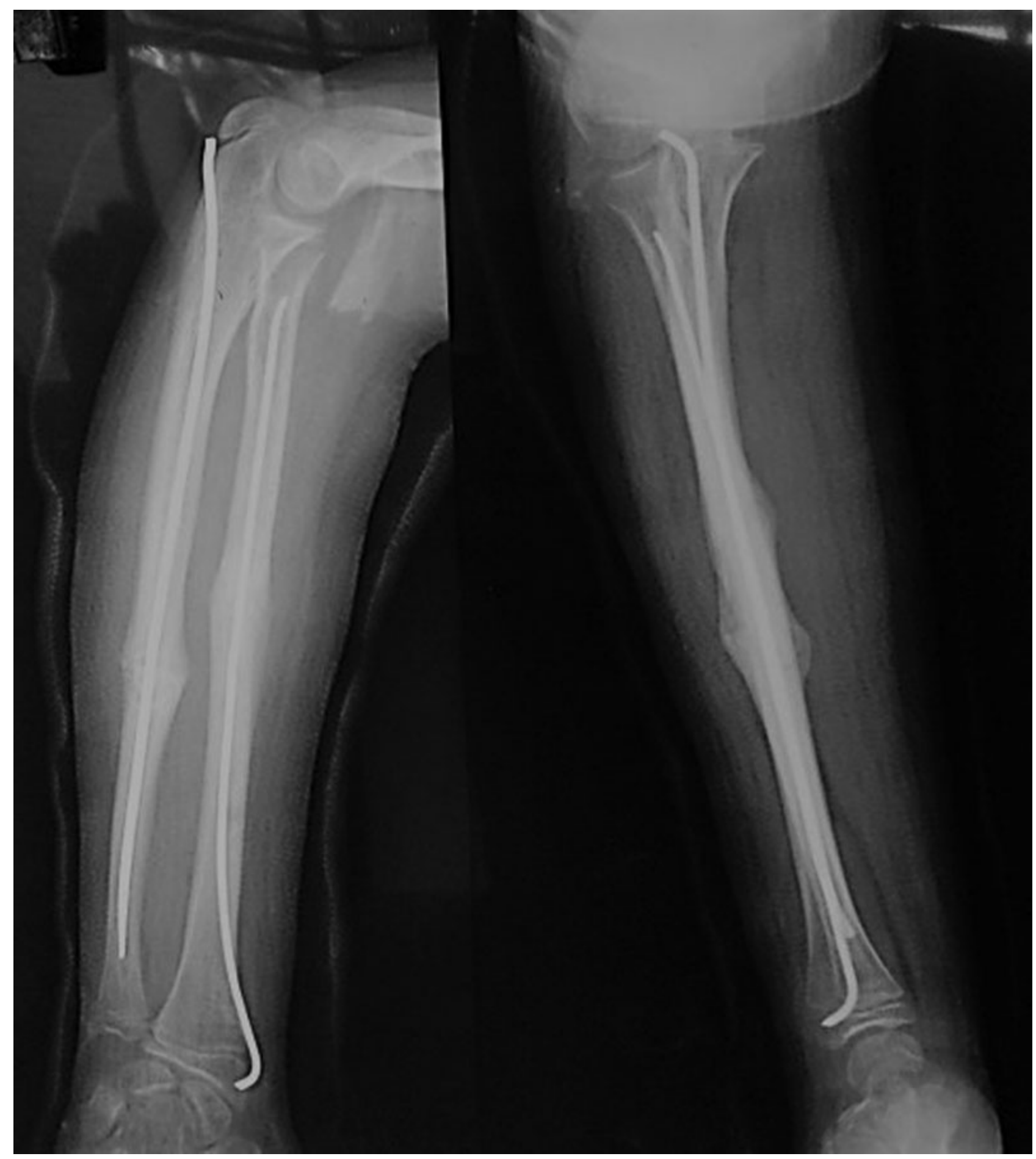

Fig. 2 Visão anteroposterior e vista lateral - a boa união da fratura da mesma criança após seis semanas da operação.

remodelam de forma previsível; portanto, estas requerem uma redução mais anatômica. ${ }^{14}$ Existe controvérsia sobre qual quantidade de angulação, deslocamento e rotação constitui uma redução aceitável. Pacientes mais jovens podem tolerar mais deformidades do que crianças mais velhas. ${ }^{16-18}$

No presente estudo, a maioria das crianças era do sexo masculino e sofreu fraturas no lado direito, semelhante a outros estudos. ${ }^{14,19}$ A média de idade dos pacientes foi de 12,9 anos em nosso estudo; Flynn relatou 10,6 anos, e Yalcinkaya relatou 10 anos. $^{14,19}$
A abertura mínima do local da fratura durante a redução foi necessária em $32,23 \%$ dos casos; Parajuli et al ${ }^{20}$ relataram que $38 \%$ dos casos necessitaram de redução aberta, e Borges et $\mathrm{a}^{21}$ relataram que 13,33\% dos casos necessitaram de redução aberta. O período médio de acompanhamento em nosso estudo foi de 8,5 meses, e o tempo médio de união foi de 7,9 semanas. Yalcinkaya et al $^{19}$ reportaram entre 6 e 8 semanas, e Flynn et $\mathrm{al}^{14}$ reportaram entre 6,9 e 8 semanas, resultados semelhantes aos do nosso estudo. Utilizamos os critérios de Price para a avaliação funcional e obtivemos 93,55\% de 
excelentes resultados e $6,45 \%$ de bons resultados. Outros também relataram resultados semelhantes. ${ }^{13,19,20}$ Shoemaker et $\mathrm{al}^{13}$ relataram $96,8 \%$; Yalcinkaya et $\mathrm{al}^{19}$ relataram $82,2 \%$, e Parajuli et $\mathrm{al}^{20}$ relataram $94 \%$ de resultados excelentes, respectivamente, usando os critérios de Price. Até agora, a remoção de implantes foi feita em uma média de 7 meses após a operação; Parajuli ${ }^{20}$ relatou a remoção de implantes seis meses após a operação, o que é comparável ao nosso estudo.

Os parâmetros aceitáveis de desalinhamento rotacional variam de $30^{\circ}$ a $45^{\circ}$ a nenhum, e alguns autores observaram que o remodelamento rotacional não é previsível. ${ }^{2,13,18,22}$ Daruwalla $^{23}$ recomendou intervenção cirúrgica para fraturas diafisárias e proximais do antebraço com angulações $>10^{\circ}$ devido ao limitado potencial de remodelação nestas áreas do osso. Deformidades residuais podem afetar o movimento do antebraço. Mathews et al mostraram em um estudo com cadáveres que as deformidades angulares do antebraço de $10^{\circ}$ não resultarão em perda significativa da pronação/supinação do antebraço, mas que uma angulação de $20^{\circ}$ restringirá a rotação do antebraço em $\sim 30 \%{ }^{24}$ Em outro estudo com cadáveres, Tarr et $\mathrm{al}^{25}$ demonstraram que a angulação da fratura diafisária entre $5^{\circ}$ e $10^{\circ}$ graus no antebraço pode levar a um déficit de pronação de 5 a $27 \%$. Dado o potencial fracasso da solução não cirúrgica (de 1.5 a 31\%) e a importância de minimizar a deformidade angular para preservar a rotação normal do antebraço, o manejo cirúrgico da fratura pediátrica do antebraço tem sido cada vez mais popular. ${ }^{25}$

Quando indicada, a fixação cirúrgica de fraturas pediátricas do antebraço geralmente é eficaz, independentemente do método de fixação. ${ }^{13,14,22,24,26}$ A haste IM flexível é o método de fixação preferido para fraturas pediátricas do antebraço. A maioria das séries mostra resultados de bons a excelentes usando este método. ${ }^{13,14,19,24,26}$ Tanto a redução fechada quanto a redução aberta antes da inserção da haste IM produziram resultados funcionais semelhantes, com perfil de complicação semelhante na fratura diafisária pediátrica. ${ }^{19}$ No presente estudo, 10 pacientes necessitaram de uma abertura mínima do local da fratura durante a redução devido à interposição de tecidos moles ou de canulação difícil devido à formação de calo. Embora não tenhamos comparado os resultados da técnica fechada versus a aberta, incluímos as duas técnicas, sendo os resultados de bons a excelentes.

As complicações do presente estudo são comparáveis às de outros vários estudos. Yalcinkaya et al relatou uma taxa de complicações variando de 4 a $38 \%$ em pacientes tratados com haste IM, e Flynn et al mostrou que a taxa geral de complicações em pacientes submetidos a haste IM era de $14,6 \%{ }^{14,19}$ As complicações mais comuns que ocorreram em suas séries foram atraso na consolidação óssea, síndrome compartimental, infecção, irritação da pele pelo material, e recuo do pino. Em nossa série, complicações menores foram notadas em 5 (16,12\%) pacientes. Não ocorreram ausência de consolidações ou consolidações mal realizadas, e não houve infecções profundas comparáveis com as de outros estudos. 13,14,19,20

Uma limitação do presente estudo é sua natureza não comparativa. Um estudo semelhante com um grupo controle não operatório ou um estudo comparativo com outra técnica operatória com um acompanhamento de maior duração seria ideal para uma conclusão definitiva.

\section{Conclusão}

Hastes flexíveis são uma solução versátil e eficiente para fixação interna de fraturas diafisárias de ambos os ossos do antebraço em crianças, permitindo mobilização e consolidação precoces, excelente resultado funcional e complicações gerenciáveis. É uma alternativa válida para o tratamento de deslocamento da diáfise em fraturas de ambos os ossos do antebraço em crianças.

Conflitos de Interesses

Os autores declaram não haver conflitos de interesses.

\section{Referências}

1 Beaty JH, Kasser JR. Rockwood and Wilkins fractures in children. 7th ed. Philadelphia: Lippincott Williams \& Wilkins; 2010

2 Smith VA, Goodman HJ, Strongwater A, Smith B. Treatment of pediatric both-bone forearm fractures: a comparison of operative techniques. J Pediatr Orthop 2005;25(03):309-313

3 Bowman EN, Mehlman CT, Lindsell CJ, Tamai J. Nonoperative treatment of both-bone forearm shaft fractures in children: predictors of early radiographic failure. J Pediatr Orthop 2011;31(01):23-32

4 Reinhardt KR, Feldman DS, Green DW, Sala DA, Widmann RF, Scher DM. Comparison of intramedullary nailing to plating for both-bone forearm fractures in older children. J Pediatr Orthop 2008;28(04):403-409

5 Volpon JB. Osteossíntese das fraturas diafisárias da criança com hastes intramedulares flexíveis. Rev Bras Ortop 2008;43(07):261-270

6 Alvachian Fernandes HJ, Saad EA, Reis FB. Osteosynthesis with intramedullary nails in children. Rev Bras Ortop 2015;44(05): 380-385

7 Westacott DJ, Jordan RW, Cooke SJ. Functional outcome following intramedullary nailing or plate and screw fixation of paediatric diaphyseal forearm fractures: a systematic review. J Child Orthop 2012;6(01):75-80

8 Wall L, O'Donnell JC, Schoenecker PL, et al. Titanium elastic nailing radius and ulna fractures in adolescents. J Pediatr Orthop B 2012; 21(05):482-488

9 Barry M, Paterson JM. A flexible intramedullary nails for fractures in children. J Bone Joint Surg Br 2004;86(07):947-953

10 Gustilo RB, Anderson JT. Prevention of infection in the treatment of one thousand and twenty-five open fractures of long bones: retrospective and prospective analyses. J Bone Joint Surg Am 1976;58(04):453-458

11 Price CT, Scott DS, Kurzner ME, Flynn JC. Malunited forearm fractures in children. J Pediatr Orthop 1990;10(06):705-712

12 Lascombes P, Prevot J, Ligier JN, Metaizeau JP, Poncelet T. Elastic stable intramedullary nailing in forearm shaft fractures in children: 85 cases. J Pediatr Orthop 1990;10(02):167-171

13 Shoemaker SD, Comstock CP, Mubarak SJ, Wenger DR, Chambers HG. Intramedullary Kirschner wire fixation of open or unstable forearm fractures in children. J Pediatr Orthop 1999;19(03): 329-337

14 Flynn JM, Jones KJ, Garner MR, Goebel J. Eleven years experience in the operative management of pediatric forearm fractures. J Pediatr Orthop 2010;30(04):313-319

15 Rodríguez-Merchán EC. Pediatric fractures of the forearm. Clin Orthop Relat Res 2005;(432):65-72

16 Wilkins KE. Operative management of children's fractures: is it a sign of impetuousness or do the children really benefit? J Pediatr Orthop 1998;18(01):1-3 
17 Schmittenbecher PP. State-of-the-art treatment of forearm shaft fractures. Injury 2005;36(Suppl 1):A25-A34

18 Teoh KH, Chee YH, Shortt N, Wilkinson G, Porter DE. An age- and sex-matched comparative study on both-bone diaphyseal paediatric forearm fracture. J Child Orthop 2009;3(05):367-373

19 Yalçinkaya M, Doğan A, Ozkaya U, Sökücü S, Uzümcügil O, Kabukçuoğlu Y. Clinical results of intramedullary nailing following closed or mini open reduction in pediatric unstable diaphyseal forearm fractures. Acta Orthop Traumatol Turc 2010;44 (01):7-13

20 Parajuli NP, Shrestha D, Dhoju D, Dhakal GR, Shrestha R, Sharma V. Intramedullary nailing for paediatric diaphyseal forearm bone fracture. Kathmandu Univ Med J (KUMJ) 2011;9(35):198-202

21 Borges JLP, Silva VC, Penteado LH. Fixação intramedular das fraturas do antebraço em crianças. Rev Bras Ortop 1996;31(01):41-45
22 Richter D, Ostermann PA, Ekkernkamp A, Muhr G, Hahn MP. Elastic intramedullary nailing: a minimally invasive concept in the treatment of unstable forearm fractures in children. J Pediatr Orthop 1998;18(04):457-461

23 Daruwalla JS. A study of radioulnar movements following fractures of the forearm in children. Clin Orthop Relat Res 1979;(139):114-120

24 Matthews LS, Kaufer H, Garver DF, Sonstegard DA. The effect on supination-pronation of angular malalignment of fractures of both bones of the forearm. J Bone Joint Surg Am 1982;64(01):14-17

25 Tarr RR, Garfinkel AI, Sarmiento A. The effects of angular and rotational deformities of both bones of the forearm. An in vitro study. J Bone Joint Surg Am 1984;66(01):65-70

26 Garg NK, Ballal MS, Malek IA, Webster RA, Bruce CE. Use of elastic stable intramedullary nailing for treating unstable forearm fractures in children. J Trauma 2008;65(01):109-115 